\title{
Optimizing neurodevelopmental outcomes in infants and children following prenatal SSRI exposure
}

\author{
"It is possible that SSRI exposure and maternal mood interact with \\ genetic factors to affect levels of serotonin during development, which \\ could influence disease susceptibility later in life via altered early levels of \\ central 5-HT, suggesting 'fetal serotonergic programming'."
}

A week does not go by without new studies reporting neonatal and infant outcomes following prenatal selective serotonin-reuptake inhibitor (SSRI) antidepressant exposure. Recent attention has focused on an increased risk for congenital anomalies - particularly cardiac defects [1] - and neonatal neurobehavioral disturbances associated with prenatal SSRI exposure [2]. Other studies reported no increase in long-term developmental vulnerability [3], often leaving scientific [4] and public [5,101] communities concerned about how, when or even whether SSRIs should be used during pregnancy [6].

As a developmental pediatrician, I am frequently faced with questions about how best to promote optimal development and behavior in children whose mothers were depressed during pregnancy. Antenatal maternal mood disorders themselves influence infant and child behavior and so we are often asked how to provide appropriate care where both antenatal SSRI and maternal mood effects are frequently indistinguishable $[7,8]$. To appreciate the impact of prenatal SSRI exposure, we need to recognize the interplay of three key features influencing child development in this setting. This is a story of the 'twos': two environmental pathogens (exposure to depressed/anxious maternal mood and SSRI antidepressants); two time periods(spanning pre- and post-natal periods); and two highly interrelated individuals (the mother and her infant). Accounting for the ongoing interplay between these factors is essential, but our current understanding of the impact of SSRI medications on early human brain development limits our ability to distinguish between the impact of disease and treatment. Both mood disturbances and SSRI treatment during pregnancy are increasingly common. Exposure to maternal depression during gestation occurs in $10-20 \%$ of pregnancies and up to a third of all depressed mothers have been reported to be treated with an
SSRI during pregnancy [9]. Since SSRIs have not been reported to produce gross structural neuroteratogenic effects, they are often considered for antenatal therapy [10], with the expectation that they optimize maternal mental health during pregnancy. However, since both a mother's mood disturbances (and inherent counfounding fractors such as smoking, alcohol and socieconomic factors) and SSRI treatment during pregnancy can have long-lasting consequences on cognitive and emotional child development [11], mothers and clinicians must often weigh potential consequences of untreated mental illness against consequences of antenatal psychopharmacotherapy.

“Prenatal changes in central neurotransmitters during periods of fetal development may lead to subtle functional effects and CNS vulnerability, extending into childhood and potentially across the lifespan."

Understanding how antenatal maternal mood disturbances and SSRI antidepressants influence early brain development can provide us with critical clues as to how early adverse life experiences program developing neural systems in ways that might contribute to health risks, such as depression, later in life. Both prenatal maternal mood disturbances and SSRIs affect levels of the key neurotransmitter serotonin $(5-\mathrm{HT})$ during critical periods of neurodevelopment. 5-HT plays two critical roles; first, during early developmental periods 5-HT acts as a growth factor, regulating the development of its own and related neural systems [12]. Second, later in development it works as a neurotransmitter regulating cognition, attention, emotion, learning, sleep, arousal and stress responsivity. SSRIs
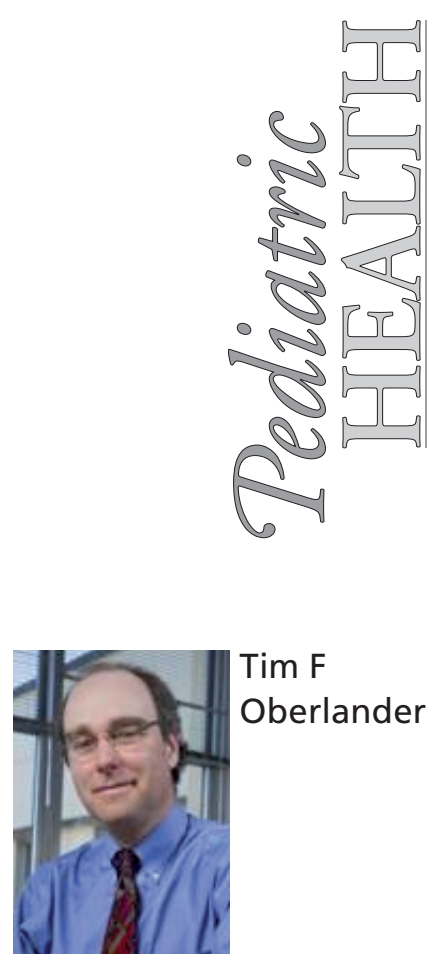

Early Human Experience Unit, Child \& Family Research Institute, Room L408, 4480 Oak St., Vancouver BC V6 3V4, Canada Tel.: +1 6048753570

Fax: +1 6048753569

toberlander@cw.bc.ca

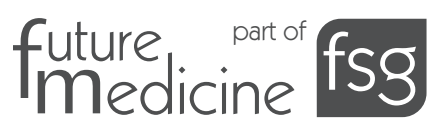


work primarily by blocking the 5-HT transporter leading to increased extracellular 5-HT levels, and since SSRIs readily cross the placenta and the blood-brain barrier [13], prenatal SSRI treatment alters fetal central 5-HT levels. Therefore, it is conceivable that altering levels of 5-HT secondary to SSRI exposure might change brain development and subsequent 5-HT-related behaviors. Until recently, there has been a paucity of similar evidence in humans, but evidence is emerging suggesting that there are sustained neurodevelopmental effects [11,14]. To date, no gross SSRI-related neuroteratogenic effects have been identified, yet evidence of subtle functional behavioral disturbances associated with fetal SSRI exposure have been reported [2]. Some outcomes reflect a 'main effect' for the SSRI exposure [15], but beyond infancy, childhood development appears typical [3] or is predicted by ongoing maternal mood disturbances [16]. Neonatal behaviors may be associated with cord drug levels and neurotransmitter alterations [17], and genetic variables also play a moderating role, although not all alleles carry the same risk [18]. Why some children are affected by prenatal SSRI exposure and others are not remains a pressing question. It is possible that SSRI exposure and maternal mood interact with genetic factors to affect levels of serotonin during development $[17,18]$, which could influence disease susceptibility later in life via altered early levels of central 5-HT, suggesting 'fetal serotonergic programming' [11].

“...affected behaviors of interest may only emerge with time as they may be masked (or sleeper) effects in yet-to-emerge capacities ... such as executive functions that regulate attention and arousal and only come 'online' when a child reaches the developmental stage where the capacity is required."

In our search for an understanding of the impact of prenatal mood disturbances or medication exposure, we need to apply a broader developmental 'lens' through which to examine childhood behavior. Prenatal changes in central neurotransmitters during periods of fetal development may lead to subtle functional effects and CNS vulnerability, extending into childhood and potentially across the lifespan. As 5-HT regulates arousal, memory, mood and attention, alterations in such behaviors may not be readily apparent at birth. In this sense, the altered developing neural system function may only manifest in varying ways owing to the changing functions or demands on the system [19]. With this perspective, affected behaviors of interest may only emerge with time as they may be masked (or sleeper) effects in yetto-emerge capacities [19]. This is particularly true of domains such as executive functions that regulate attention and arousal and only come 'online' when a child reaches the developmental stage where the capacity is required. Moreover, as a potential behavioral teratogen, the effects of SSRI exposure may only affect functioning in a temporary or transitory matter. As a case in point, neonatal withdrawal symptoms may only appear for a short time, then disappear, but could subsequently be antecedent to other altered behaviors later in childhood [20].

"From a child-health perspective, our task is to recognize risks arising from both the maternal disease and its treatment, and our challenge is to find ways to 'stack the deck' in favor of healthy and optimal childhood development and behavior."

In summarizing our current knowledge, three themes emerge. First, this is not a 'main effects' story where outcomes can easily be attributed to one causal factor (i.e., maternal mood or the drug), but rather outcomes in this setting represent an interplay of psychological, pharmacological, genetic and social factors related to both mother and child. This is indeed a setting where nature meets nurture and niche [21]. Second, while SSRIs might be prescribed during pregnancy, with the expectation of optimizing infant health following improved maternal mood, children may continue to be at risk as maternal pharmacotherapy might not 'buffer' or protect them from antenatal or postnatal maternal mood disturbances. Third, in this context of developmental vulnerability and resilience, identifying children who could benefit from prenatal maternal SSRI treatment remains a pressing and unanswered question. Longitudinal study designs that integrate a maternal and infant/child developmental perspective should help us move away from regarding maternal mood disturbances, prenatal SSRI exposure or even specific genotypes as 'bad' or 'harmful' and rather look at these as adversity or risk-related factors that heighten or lessen vulnerability given a particular life experience. 
From a child-health perspective, our task is to recognize risks arising from both the maternal disease and its treatment. Our challenge is to find ways to 'stack the deck' in favor of healthy and optimal childhood development and behavior, in the context of family wellbeing. While the decision to initiate SSRI treatment during pregnancy rests with the mother and her physician carefully weighing the risks and benefits [22], we play a critical role in this setting; as pediatricians we may be the earliest point of contact that first identifies a depressed or anxious new mother. In providing care in this setting we need to recognize risk characteristics that are inherent to the individual child - in contrast to seeing them as just part of a population of prenatally exposed children. Care should focus on effectively addressing maternal and infant needs with all available pharmacological and nonpharmacological options (cognitive/ behavioral, social support, diet, housing etc.), remembering that medications may be just one of many options available. This should include addressing the wellbeing of the entire family and its social context, ensuring access to affordable and appropriate healthcare, and providing lay community support. Addressing barriers to identifying and treating maternal perinatal mood disturbances should be seen as an urgent public health concern that will benefit mothers and children alike.
Ultimately, it might not be possible to distinguish the effects of disease from treatment, nor may it even be necessary. What is critical is that we recognize that multiple and ongoing 'environmental pathogens' in this setting require watchful surveillance and timely interventions. The needs of mothers and their children in this setting may differ, but the wellbeing of each is critical to the other. Recognizing inter-related risks associated with both antenatal maternal mood disorders and SSRI exposure is critical for developing empirical, evidence-based approaches that identify the best fit between a pharmacological agent, nonpharmacological therapy, and maternal and neonatal factors so as to balance risks and benefits for both mothers and their children. This is a fascinating but complex story that has much to teach us about the basic science of child development and offers many clues that should help promote healthy child development.

\section{Financial \& competing interests disclosure}

Tim F Oberlander is supported by a HELP Senior Career Award and is the R Howard Webster Professorship in Child Development (College of Interdisciplinary Studies, UBC). The author has no other relevant affliations or financial involvement with any organization or entity with a financial interest in or financial conflict with the subject matter or materials discussed in the manuscript apart from those disclosed.

The author is grateful to Ursula Brain and Katherine Wisner for their thoughtful comments.

\section{Bibliography}

1. Pedersen LH, Henriksen TB, Vestergaard M et al.: Selective serotonin reuptake inhibitors in pregnancy and congenital malformations: population based cohort study BMJ 339, b3569 (2009).

2. Zeskind, PS, Stephens LE: Maternal selective serotonin reuptake inhibitor use during pregnancy and newborn neurobehavior Pediatrics 113(2), 368-375 (2004).

3. Nulman I, Rovet J, Stewart DE et al.: Neurodevelopment of children exposed in utero to antidepressant drugs. N. Engl. J. Med. 336(4), 258-262 (1997).

4. Einarson A, Schachtschneider AK, Halil R, Bollano E, Koren G: SSRIs and other antidepressant use during pregnancy and potential neonatal adverse effects: impact of a public health advisory and subsequent reports in the news media. BMC Pregnancy Childbirth 5(1), 11 (2005).

5. Jetter A: Pregnant pause. Vogue 144-232 (2009).
6. Yonkers KA: Parsing risk for the use of selective serotonin reuptake inhibitors in pregnancy. Am. J. Psychiatry 166(3), 268-270 (2009).

7. O'Connor TG, Heron J, Golding J, Beveridge M, Glover V: Maternal antenatal anxiety and children's behavioural/emotional problems at 4 years. Report from the Avon Longitudinal Study of Parents and Children. Br. J. Psychiatry 180, 502-508 (2002).

8. Talge NM, Neal C, Glover V: Antenatal maternal stress and long-term effects on child neurodevelopment: how and why? J. Child Psychol. Psychiatry 48(3-4), 245-261 (2007).

9. Oberlander TF, Warburton W, Misri S, Aghajanian J, Hertzman C: Neonatal outcomes after prenatal exposure to selective serotonin reuptake inhibitor antidepressants and maternal depression using populationbased linked health data. Arch. Gen. Psychiatry 63(8), 898-906 (2006).

10. Gentile S: SSRIs in pregnancy and lactation: emphasis on neurodevelopmental outcome. CNS Drugs 19(7), 623-633 (2005).
11. Oberlander TF, Gingrich JA, Ansorge MS: Sustained neurobehavioral effects of exposure to SSRI antidepressants during development: molecular to clinical evidence. Clin. Pharmacol. Ther. DOI:10.1038/clpt.2009.201 (2009) (Epub ahead of print).

12. Whitaker-Azmitia PM, Druse M, Walker P, Lauder JM: Serotonin as a developmental signal. Behav. Brain Res. 73(1-2), 19-29 (1996).

13. Kim J, Riggs KW, Misri S et al.: Stereoselective disposition of fluoxetine and norfluoxetine during pregnancy and breast-feeding. Br. J. Clin. Pharmacol. 61(2), 155-163 (2006).

14. Ansorge MS, Hen R, Gingrich JA: Neurodevelopmental origins of depressive disorders. Curr. Opin. Pharmacol. 7(1), 8-17 (2007).

15. Moses-Kolko EL, Bogen D, Perel J et al.: Neonatal signs after late in utero exposure to serotonin reuptake inhibitors: literature review and implications for clinical applications. JAMA 293(19), 2372-2383 (2005). 
16. Misri S, Reebye P, Kendrick K et al.: Internalizing behaviors in 4-year-old children exposed in utero to psychotropic medications. Am. J. Psychiatry 163(6), 1026-1032 (2006).

17. Hilli J, Heikkinen T, Rontu R et al.: MAO-A and COMT genotypes as possible regulators of perinatal serotonergic symptoms after in utero exposure to SSRIs. Eur. Neuropsychopharmacol. 19(5), 363-370 (2009).

18. Oberlander TF, Bonaguro RJ, Misri S, Papsdorf M, Ross CJ, Simpson EM: Infant serotonin transporter (SLC6A4) promoter genotype is associated with adverse neonatal outcomes after prenatal exposure to serotonin reuptake inhibitor medications. Mol. Psychiatry 13(1), 65-73 (2008).

19. Fried PA: Conceptual issues in behavioral teratology and their application in determining long-term sequelae of prenatal marihuana exposure. J. Child Psychol. Psychiatry 43(1), 81-102 (2002).
20. Oberlander TF, Reebye P, Misri S, Papsdorf M, Kim J, Grunau R: Externalizing and attentional behaviors in children of depressed mothers treated with a selective serotonin reuptake inhibitor antidepressant during pregnancy. Arch. Pediatr. Adolesc. Med. 161, 22-29 (2007).

21. Eisenberg L: Nature, niche, and nurture. Acad. Psychiatry 22(4), 213-222 (1998).

22. Wisner KL, Zarin DA, Holmboe ES et al: Risk-benefit decision making for treatment of depression during pregnancy. Am. J. Psychiatry 157(12), 1933-1940 (2000).

\section{Website}

101. Harding A: Antidepressant use in pregnancy can affect newborns. (2009). www.cnn.com/2009/HEALTH/10/06/ antidepressant.pregnancy.newborn/index. html?iref=newssearch

\section{Affiliation}

- Tim F Oberlander, MD, FRCPC Department of Pediatrics, University of British Columbia, Vancouver, BC, Canada and

Child \& Family Research Institute, University of British Columbia, Vancouver, BC, Canada

Tel.: + 16048753570

Fax: +1 6048753569

toberlander@cw.bc.ca 